\title{
Para-Aortic Lymph Node Biopsy
}

National Cancer Institute

\section{Source}

National Cancer Institute. Para-Aortic Lymph Node Biopsy. NCI Thesaurus. Code C136289.

A surgical procedure in which a single or multiple para-aortic lymph nodes are removed for microscopic examination. 